\title{
Polypropylene/clay nanocomposites: mechanical response, damage, and fracture
}

\author{
A.D. Drozdov ${ }^{1, a}$, J.deC. Christiansen ${ }^{2}$, and A.-L. Høg Lejre ${ }^{1}$ \\ 1 Danish Technological Institute, 2630 Taastrup, Denmark \\ 2 Department of Production, Aalborg University, 9220 Aalborg, Denmark
}

\section{Introduction}

This study deals with experimental investigation and constitutive modeling of the mechanical behavior, damage, and fracture of polymer/clay hybrids. The attention focuses on unexpected features of their viscoplastic responses, damage accumulation, and crack propagation.

\section{Preparation of nanocomposites}

Nanocomposites were manufactured by using isotactic polypropylene Moplen HP 400R (Albis Plastic Scandinavia AB, Sweden) as a matrix, organically modified montmorillonite nanoclay Delitte $67 \mathrm{G}$ (Laviosa Chimica Mineraria S.p.A., Italy) as a filler, and maleic anhydride grafted polypropylene Eastman G 3015 (Eastman Chemical Company, USA) as a compatibilizer. Hybrids were prepared by using a two-step melt mixing process: (i) masterbatches with various clay/compatibilizer proportions were extruded in a twin-screw extruder Brabender PL2000, and (ii) neat polypropylene and the masterbatches were melt mixed at various proportions (corresponding to various concentrations of nanoclay) at molding of dumbbell specimens in injection-molding machine Arburg 320C. Dispersion of clay was examined by means of rheological tests (small-amplitude shear oscillations). The graphs of storage and loss moduli demonstrated strong low-frequency plateaus conventionally associated with good exfoliation of nanofiller.

\section{Tensile tests}

The effects of clay content and clay/compatibilizer proportion on the mechanical response of nanocomposites was analyzed in uniaxial tensile tests. Experiments were conducted by means of universal testing machine Instron-5568 equipped with a thermal chamber at various strain rates (ranging from $\dot{\epsilon}=2 \cdot 10^{-4}$ to $10^{-1} \mathrm{~s}^{-1}$ ) and various temperatures $T$ (in the interval from room temperature to $100{ }^{\circ} \mathrm{C}$ ). Observation demonstrate noticeable improvement of mechanical properties (Young's modulus and yield stress) of polypropylene induced by its reinforcement. As unexpected results, it is worth mentioning (i) a rather low critical concentration of nanoclay (about 1-2 wt.\%), (ii) weak influence of clay/compatibilizer ratio on the response of hybrids, and (iii) enhancement of reinforcement at elevated temperatures (the growth of yield stress due to presence of nanofiller at $T=70{ }^{\circ} \mathrm{C}$ exceeds that at room temperature by twice).

a e-mail: Aleksey.Drozdov@teknologisk.dk 


\section{Volume growth under stretching}

To assess volume growth in nanocomposites under stretching, uniaxial tensile tests were conducted, where longitudinal and transverse strains were monitored simultaneously. Experimental data show that the entire interval of tensile strains $\epsilon$ may be split into 3 sub-intervals: (i) in the domain of linear elasticity $(\epsilon<0.015)$, Poisson's ratio $\nu_{1}$ is close to 0.4 and practically independent of clay content $\chi$, (ii) in the sub-yield region $\left(0.015<\epsilon<\epsilon_{\mathrm{y}}\right.$, where $\epsilon_{\mathrm{y}}$ stands for the yield strain), Poisson's ratio $\nu_{2}$ of neat polypropylene equals 0.5 , while $\nu_{2}$ of nanocomposites linearly decreases with $\chi$, (iii) in the interval of post-yield deformations $\left(\epsilon>\epsilon_{\mathrm{y}}\right)$, volume dilation in nanocomposites differ from that in neat polymer. While polypropylene remains incompressible under tension up to a close vicinity of the necking point, substantial (by 20\%) increase in volume is observed in hybrids. The growth starts at the yield point, occurs in a self-accelerating manner, and its intensity is strongly affected by clay content.

\section{Cyclic tests}

Keeping in mind that volume growth (which reflects nucleation, formation, and coalescence of micro-voids) is conventionally associated with damage, it seems natural to expect that the mechanical response of nanocomposites under cyclic deformation deteriorates with number of cycles. To validate this hypothesis, cyclic tensile tests with a strain-controlled program were conducted, where specimens were stretched up to some maximum strain $\epsilon_{1 \text { max }}$, retracted down to a tensile stress $\sigma_{\min }$, stretched up to a new strain $\epsilon_{2 \max }>\epsilon_{1 \text { max }}$, retracted down to $\sigma_{\min }$, etc. Surprisingly, nanocomposites demonstrated strong strain-hardening (Mullins' effect): when tension proceeded after cyclic loading and the current strain exceeded a maximum strain in the previous cycle, the stress-strain curve rapidly reached that for a virgin specimen. Moreover, total fading of memory about deformation history was revealed: not only loading paths of the stress-strain diagrams coincided for virgin samples and those subjected to cyclic deformation, but appropriate unloading path were indistinguishable as well.

Another unexpected result was observed in cyclic tests with a stress-controlled program (ratcheting between a maximum $\sigma_{\max }$ and a minimum $\sigma_{\min }$ stresses). As reinforcement of polypropylene with clay leads to rather modest growth (15-30\%) of yield stress $\sigma_{\mathrm{y}}$, one could presume the same level of decay in residual strain. Surprisingly, experimental data showed a pronounced (by several times) reduction in residual strain in low-cycle (30-100) fatigue tests after reinforcement of polypropylene with $1 \mathrm{wt} . \%$ of nanofiller only.

\section{Essential work of fracture}

To assess fracture toughness of nanocomposites, tensile tests were conducted on DENT (doubleend notched) specimens, where tensile strain was measured far away from the ligament. Experiments were performed with various strain rates at various temperatures. The data were employed to construct stress-strain diagrams under loading and unloading (driven by rupture of samples), to evaluate the energy stored in broken samples due to viscoplastic deformation, and to account for it in the energy balance law. Observations show (i) a pronounced increase (by twice) in specific essential work of fracture with clay content, (ii) a strong dependence of the rate of crack propagation on $\chi$, and (iii) a weak influence of ligament width on the fracture process.

\section{Conclusions}

Experimental data are reported on polypropylene/clay nanocomposites in mechanical and fracture tests. Constitutive models are derived for the description of observed phenomena, and their adjustable parameters are found by fitting the data. Structure-property relations are discussed for the hybrids. 